\title{
ADMISSIBLE WAVELETS ASSOCIATED WITH THE HEISENBERG GROUP
}

\author{
Heping LiU and Lizhong Peng
}

Let NAK be the Iwasawa decomposition of group SU $(n+$ $1,1)$. The Iwasawa subgroup $\mathbf{P}=\mathbf{N A}$ can be identified with the generalized upper half-plane $\mathbf{U}^{n+1}$ and has a natural representation $U$ on the $L^{2}$-space of the Heisenberg group $L^{2}\left(\mathbf{H}^{n}\right)$. We decompose $L^{2}\left(\mathbf{H}^{n}\right)$ into the direct sum of the irreducible invariant closed subspaces under $U$. The restrictions of $U$ on these subspaces are square-integrable. We characterize the admissible condition in terms of the Fourier transform and define the wavelet transform with respect to admissible wavelets. The wavelet transform leads to isometric operators from the irreducible invariant closed subspaces of $L^{2}\left(\mathbf{H}^{n}\right)$ to $L^{2, \nu}\left(\mathbf{U}^{n+1}\right)$, the weighted $L^{2}$-spaces on $\mathbf{U}^{n+1}$. By selecting a set of mutual orthogonal admissible wavelets, we get the direct sum decomposition of $L^{2, \nu}\left(\mathbf{U}^{n+1}\right)$ with the first component $A^{\nu}\left(\mathbf{U}^{n+1}\right)$, the (weighted) Bergman space.

\section{Introduction.}

First let us recall the admissible wavelets and decomposition of function spaces associated with the group $\mathbf{S L}(2, \mathbf{R})$ (cf. [5], [7], [8] and [13]). Let $\mathbf{U}=\{z \in \mathbf{C}: \operatorname{Im} z>0\}$ be the upper half-plane. $\mathbf{S L}(2, \mathbf{R})$ acts on $\mathbf{U}$ by means of maps

$$
T_{g}: z \longmapsto g z=\frac{a z+b}{c z+d}, \quad z \in \mathbf{U}, g=\left(\begin{array}{ll}
a & b \\
c & d
\end{array}\right) \in \mathbf{S L}(2, \mathbf{R}) .
$$

Let $\mathbf{S L}(2, \mathbf{R})=\mathbf{N A K}$ be the Iwasawa decomposition, where

$$
\begin{aligned}
& \mathbf{N}=\left\{b=\left(\begin{array}{ll}
1 & b \\
0 & 1
\end{array}\right): \quad b \in \mathbf{R}\right\}, \\
& \mathbf{A}=\left\{a=\left(\begin{array}{cc}
\sqrt{a} & 0 \\
0 & \frac{1}{\sqrt{a}}
\end{array}\right): \quad a \in \mathbf{R}^{+}\right\}, \\
& \mathbf{K}=\left\{\left(\begin{array}{cc}
\cos \theta \sin \theta \\
-\sin \theta & \cos \theta
\end{array}\right): \quad \theta \in[0,2 \pi)\right\} .
\end{aligned}
$$


Let

$$
\mathbf{P}=\mathbf{N} \mathbf{A}=\left\{(b, a)=\left(\begin{array}{cc}
\sqrt{a} & \frac{b}{\sqrt{a}} \\
0 & \frac{1}{\sqrt{a}}
\end{array}\right): \quad b \in \mathbf{R}, a \in \mathbf{R}^{+}\right\}
$$

$\mathbf{P}$ is a solvable subgroup of $\mathbf{S L}(2, \mathbf{R})$. As subgroups of $\mathbf{S L}(2, \mathbf{R}), \mathbf{N}$ acts on $\mathbf{U}$ by the translations with respect to $\partial \mathbf{U}=\mathbf{R}$

$$
T_{b}: z \longmapsto z+b, \quad z \in \mathbf{U}, b \in \mathbf{R},
$$

and $\mathbf{A}$ acts on $\mathbf{U}$ by the delations

$$
T_{a}: z \longmapsto a z, \quad z \in \mathbf{U}, a \in \mathbf{R}^{+} .
$$

Obviously $\mathbf{N}$ and $\mathbf{A}$ (therefore $\mathbf{P}$ ) act on $\partial \mathbf{U}=\mathbf{R}$ also. Because $\mathbf{P}$ acts on $\partial \mathbf{U}=\mathbf{R}$ by

$$
T_{(b, a)}: x \longmapsto a x+b, \quad x \in \mathbf{R},(b, a) \in \mathbf{P},
$$

$\mathbf{P}$ coincides with the affine group " $a x+b$ ". The group law of $\mathbf{P}$ is given by

$$
(b, a)\left(b^{\prime}, a^{\prime}\right)=\left(a b^{\prime}+b, a a^{\prime}\right) .
$$

$\mathbf{P}$ is a locally compact nonunimodular group with the left Haar measure $d \mu_{l}(b, a)=\frac{d b d a}{a^{2}}$ and the right Haar measure $d \mu_{r}(b, a)=\frac{d b d a}{a}$.

Define the unitary representation $U$ of $\mathbf{P}$ on $L^{2}(\mathbf{R})$ by

$$
U_{(b, a)} f(x)=\frac{1}{\sqrt{a}} f\left(\frac{x-b}{a}\right), \quad f \in L^{2}(\mathbf{R}),(b, a) \in \mathbf{P} .
$$

$U$ is reducible on $L^{2}(\mathbf{R})$ with two irreducible invariant closed subspaces $H^{2}(\mathbf{R})$ (Hardy space) and $\overline{H^{2}}(\mathbf{R})$ (conjugate Hardy space). The restriction of $U$ on $H^{2}(\mathbf{R})$ (or $\overline{H^{2}}(\mathbf{R})$ ) is square-integrable in the sense of following: There exists in $H^{2}(\mathbf{R})$ (or $\overline{H^{2}}(\mathbf{R})$ ) at least one function $\phi$, not identically zero, such that

$$
\frac{1}{\|\phi\|_{L^{2}(\mathbf{R})}^{2}} \int_{\mathbf{P}}\left|\left\langle\phi, U_{(b, a)} \phi\right\rangle_{L^{2}(\mathbf{R})}\right|^{2} \frac{d b d a}{a^{2}}=C_{\phi}<+\infty .
$$

(1.2) is called the admissible condition, which is equivalent to

$$
C_{\phi}=\int_{-\infty}^{+\infty} \frac{|\widehat{\phi}(\xi)|^{2}}{|\xi|} d \xi<+\infty
$$

We say that a function $\phi$ is an admissible wavelet if $\phi \in H^{2}(\mathbf{R})\left(\right.$ or $\left.\overline{H^{2}}(\mathbf{R})\right), \phi$ is not identically zero and satisfies the admissible condition. Let $\phi \in H^{2}(\mathbf{R})$ 
(or $\overline{H^{2}}(\mathbf{R})$ ) be an admissible wavelet, we define the operator $W_{\phi}$ on $H^{2}(\mathbf{R})$ (or $\overline{H^{2}}(\mathbf{R})$ ) by

$$
W_{\phi} f(b, a)=C_{\phi}^{-\frac{1}{2}}\left\langle f, U_{(b, a)} \phi\right\rangle_{L^{2}(\mathbf{R})} .
$$

The function $W_{\phi} f(b, a)$ is called the (continuous) wavelet transform of $f$ with respect to $\phi$. The wavelet transform $W_{\phi} f$ is an isometric operator from $H^{2}(\mathbf{R})\left(\right.$ or $\left.\overline{H^{2}}(\mathbf{R})\right)$ into $L^{2}\left(\mathbf{P}, d \mu_{l}\right)$, i.e.,

$$
\int_{\mathbf{P}}\left|W_{\phi} f(b, a)\right|^{2} \frac{d b d a}{a^{2}}=\|f\|_{L^{2}(\mathbf{R})}^{2} .
$$

As a consequence, we have the Calderón reproducing formula

$$
f(x)=C_{\phi}^{-\frac{1}{2}} \int_{\mathbf{P}} W_{\phi} f(b, a) U_{(b, a)} \phi(x) \frac{d b d a}{a^{2}} .
$$

Moreover, we can identify $\mathbf{P}$ with $\mathbf{U}$ by setting $z=b+i a$. Let $L^{2, \nu}(\mathbf{U})=$ $\left\{f:\|f\|^{2}=\int_{\mathbf{U}}|f(z)|^{2}(\operatorname{Im} z)^{\nu} d z<+\infty\right\}$, where $d z$ denotes the Lebesgue measure. Especially $L^{2}\left(\mathbf{P}, d \mu_{l}\right)=L^{2,-2}(\mathbf{U})$. For $\nu>-1$, let $A^{\nu}(\mathbf{U})$ denote the (weighted) Bergman space, i.e., the subspace of all holomorphic functions in $L^{2, \nu}(\mathbf{U})$. If $\phi \in H^{2}(\mathbf{R})$ (or $\overline{H^{2}}(\mathbf{R})$ ) is an admissible wavelet, then the map

$$
f \longmapsto a^{-\frac{\nu+2}{2}} W_{\phi} f(b, a)
$$

is an isometric operator from $H^{2}(\mathbf{R})$ ( or $\overline{H^{2}}(\mathbf{R})$ ) into $L^{2, \nu}(\mathbf{U})$. By selectting admissible wavelets $\phi_{k} \in H^{2}(\mathbf{R})$ and $\bar{\phi}_{k} \in \overline{H^{2}}(\mathbf{R})$ suitably, we can get the direct sum decomposition of $L^{2, \nu}(\mathbf{U})$, i.e., $L^{2, \nu}(\mathbf{U})=\bigoplus_{k=0}^{+\infty}\left(A_{k} \bigoplus \bar{A}_{k}\right)$. Moreover, $A_{0}=A^{\nu}(\mathbf{U})$ when $\nu>-1$.

Jiang-Peng ([9]) considered the admissible wavelets and decomposition of function spaces associated with the Sympletic group $\mathbf{S P}(n, 1)$. The goal of this paper is to consider the same problems associated with the group $\mathbf{S U}(n+1,1)$.

Let $\mathbf{U}^{n+1}$ be the generalized upper half-plane in $\mathbf{C}^{n+1}$, i.e.,

$$
\mathbf{U}^{n+1}=\left\{\left(z, z_{n+1}\right) \in \mathbf{C}^{n+1}: \operatorname{Im} z_{n+1}>|z|^{2}\right\},
$$

where $z=\left(z_{1}, \cdots, z_{n}\right) \in \mathbf{C}^{n},|z|^{2}=\sum_{j=1}^{n}\left|z_{j}\right|^{2}$. It is well-known that $\mathbf{U}^{n+1}$ is holomorphically equivalent to the unit ball $\mathbf{B}^{n+1}$ in $\mathbf{C}^{n+1}$. The Cayley transform $\Gamma$ from $\mathbf{B}^{n+1}$ onto $\mathbf{U}^{n+1}$ is given by

$$
\left\{\begin{array}{l}
z_{j}=\frac{i w_{j}}{1-w_{n+1}}, \quad j=1, \cdots, n, \\
z_{n+1}=i \frac{1+w_{n+1}}{1-w_{n+1}}
\end{array}\right.
$$


where $w=\left(w_{1}, \cdots, w_{n}, w_{n+1}\right) \in \mathbf{B}^{n+1}$. The inverse transform $\Gamma^{-1}$ is given by

$$
\left\{\begin{array}{l}
w_{j}=\frac{2 z_{j}}{z_{n+1}+i}, \quad j=1, \cdots, n, \\
w_{n+1}=\frac{z_{n+1}-i}{z_{n+1}+i} .
\end{array}\right.
$$

Let $I_{n}$ denote the unit matrix of order $n$. Set $I_{n+1,1}=\left(\begin{array}{cc}-I_{n+1} & 0 \\ 0 & 1\end{array}\right)$. The group $\mathbf{G}=\mathbf{S U}(n+1,1)$ consists of all $(n+2) \times(n+2)$ complex matrices $g$ such that $\operatorname{det} g=1$ and $g^{*} I_{n+1,1} g=I_{n+1,1}$, where $g^{*}$ denotes the conjugate transpose of $g$. The action of $\mathbf{G}$ on $\mathbf{B}^{n+1}$ is defined by

$$
\widetilde{T}_{g}: w \longmapsto g w=\left(\frac{A w^{t}+B}{C w^{t}+D}\right)^{t}, \quad w \in \mathbf{B}^{n+1},
$$

where $A$ is an $(n+1) \times(n+1)$ matrix, $B$ an $(n+1) \times 1$ matrix, $C$ an $1 \times(n+1)$ matrix, $D$ a complex number, $g=\left(\begin{array}{ll}A & B \\ C & D\end{array}\right) \in \mathbf{G}, w^{t}$ denotes the transpose of $w$. $\mathbf{G}$ acts also on $\mathbf{U}^{n+1}$ by means of maps $T_{g}=\Gamma \widetilde{T}_{g} \Gamma^{-1}, g \in \mathbf{G}$. Let $\mathbf{G}=\mathbf{N A K}$ be the Iwasawa decomposition, where

$$
\begin{aligned}
& \mathbf{N}=\left\{n(z, t)=\left(\begin{array}{ccc}
I_{n} & i z^{t} & -i z^{t} \\
i \bar{z} 1-\frac{|z|^{2}-i t}{2} & \frac{|z|^{2}-i t}{2} \\
i \bar{z} & -\frac{|z|^{2}-i t}{2} & 1+\frac{|z|^{2}-i t}{2}
\end{array}\right): z \in \mathbf{C}^{n}, t \in \mathbf{R}\right\}, \\
& \mathbf{A}=\left\{a(\zeta)=\left(\begin{array}{ccc}
I_{n} & 0 & 0 \\
0 & \cosh \zeta \sinh \zeta \\
0 & \sinh \zeta \cosh \zeta
\end{array}\right): \zeta \in \mathbf{R}\right\}, \\
& \mathbf{K}=\left\{\left(\begin{array}{ll}
A & 0 \\
0 & D
\end{array}\right): A \in \mathbf{U}(n+1), \operatorname{det} A \cdot D=1\right\} \text {. }
\end{aligned}
$$

Let $\mathbf{P}=\mathbf{N A}$ be the semidirect product of $\mathbf{N}$ and $\mathbf{A}$, where the action of $\mathbf{A}$ on $\mathbf{N}$ is given by

$$
a(\zeta): n(z, t) \longmapsto a(\zeta) n(z, t) a(\zeta)^{-1}=\left(e^{\zeta} z, e^{2 \zeta} t\right) .
$$

$\mathbf{P}$ is a solvable subgroup of $\mathbf{G}$.

For $\left(z, z_{n+1}\right) \in \mathbf{C}^{n+1}$, we set $t=\operatorname{Re} z_{n+1}, \rho=\operatorname{Im} z_{n+1}-|z|^{2}$, and use the coordinates $z \in \mathbf{C}^{n}, t \in \mathbf{R}, \rho \in \mathbf{R}$. In this coordinat system, $\mathbf{U}^{n+1}$ is simply the set $\left\{(z, t, \rho): z \in \mathbf{C}^{n}, t \in \mathbf{R}, \rho \in \mathbf{R}^{+}\right\}$and $\partial \mathbf{U}^{n+1}$ the set $\left\{(z, t): z \in \mathbf{C}^{n}, t \in \mathbf{R}\right\}$, where we write $(z, t)$ instead of $(z, t, 0)$. Now we 
identify $\mathbf{N}$ with $\partial \mathbf{U}^{n+1}$ in such a way that $n(z, t)$ is identified with $(z, t)$ (cf. [10]). Similarly we identify $\mathbf{P}$ with $\mathbf{U}^{n+1}$ in such a way that $n(z, t) a(\zeta)$ is identified with $(z, t, \rho)$, where $\rho=e^{2 \zeta}$.

The group law of $\mathbf{N}$ (or $\partial \mathbf{U}^{n+1}$ ) is given by

$$
(z, t)\left(z^{\prime}, t^{\prime}\right)=\left(z+z^{\prime}, t+t^{\prime}+2 \operatorname{Im} z \overline{z^{\prime}}\right)
$$

where $z \overline{z^{\prime}}=\sum_{j=1}^{n} z_{j} \overline{z_{j}^{\prime}}$. So $\mathbf{N}$ (or $\partial \mathbf{U}^{n+1}$ ) is nothing but the Heisenberg group. The Heisenberg group, denoted by $\mathbf{H}^{n}$, is a Lie group with the underlying manifold $\mathbf{C}^{n} \times \mathbf{R}$ and the multiplication (1.9). $\mathbf{H}^{n}$ is an unimodular group, whose Haar measure coincides with the Lebesgue measure of $\mathbf{C}^{n} \times \mathbf{R}$. The delations of $\mathbf{H}^{n}$ are defined by $\rho(z, t)=(\sqrt{\rho} z, \rho t), \rho>0$, which are consistent with the delations of $\mathbf{U}^{n+1}$ defined by $\rho\left(z, z_{n+1}\right)=\left(\sqrt{\rho} z, \rho z_{n+1}\right)$. The group law of $\mathbf{P}$ is given by

$$
(z, t, \rho)\left(z^{\prime}, t^{\prime}, \rho^{\prime}\right)=\left(z+\sqrt{\rho} z^{\prime}, t+\rho t^{\prime}+2 \sqrt{\rho} \operatorname{Im} z \overline{z^{\prime}}, \rho \rho^{\prime}\right) .
$$

$\mathbf{P}$ is a locally compact nonunimodular group with the left Haar measure $d \mu_{l}(z, t, \rho)=\frac{d z d t d \rho}{\rho^{n+2}}$ and the right Haar measure $d \mu_{r}(z, t, \rho)=\frac{d z d t d \rho}{\rho}$, where $d z$ denotes the Lebesgue measure of $\mathbf{C}^{n}$.

Now $\mathbf{N}$ acts on $\mathbf{U}^{n+1}$ by the translations with respect to $\partial \mathbf{U}^{n+1}=\mathbf{H}^{n}$

$T_{(z, t)}:\left(z^{\prime}, t^{\prime}, \rho^{\prime}\right) \longmapsto\left(z+z^{\prime}, t+t^{\prime}+2 \operatorname{Im} z \overline{z^{\prime}}, \rho^{\prime}\right), \quad\left(z^{\prime}, t^{\prime}, \rho^{\prime}\right) \in \mathbf{U}^{n+1},(z, t) \in \mathbf{N}$.

A acts on $\mathbf{U}^{n+1}$ by the delations

$$
\begin{aligned}
& T_{a(\zeta)}:\left(z^{\prime}, t^{\prime}, \rho^{\prime}\right) \longmapsto \rho\left(z^{\prime}, t^{\prime}, \rho^{\prime}\right)=\left(\sqrt{\rho} z^{\prime}, \rho t^{\prime}, \rho \rho^{\prime}\right), \\
& \left(z^{\prime}, t^{\prime}, \rho^{\prime}\right) \in \mathbf{U}^{n+1}, a(\zeta) \in \mathbf{A},
\end{aligned}
$$

where $\rho=e^{2 \zeta}$. So the action of $\mathbf{P}$ on $\mathbf{U}^{n+1}$ coincides with the multiplication of $\mathbf{P}$ under the identification of $\mathbf{P}$ and $\mathbf{U}^{n+1}$. Obviously $\mathbf{N}$ and $\mathbf{A}$ (therefore $\mathbf{P})$ act on $\partial \mathbf{U}^{n+1}$ also, and $\mathbf{P}$ acts on $\partial \mathbf{U}^{n+1}$ by maps

$$
\begin{aligned}
T_{(z, t, \rho)}:\left(z^{\prime}, t^{\prime}\right) \longmapsto(z, t) \rho\left(z^{\prime}, t^{\prime}\right)=(z, t)\left(\sqrt{\rho} z^{\prime}, \rho t^{\prime}\right) & \\
& \left(z^{\prime}, t^{\prime}\right) \in \partial \mathbf{U}^{n+1},(z, t, \rho) \in \mathbf{P} .
\end{aligned}
$$

We see that the action of $\mathbf{P}$ on $\partial \mathbf{U}^{n+1}\left(\right.$ or $\mathbf{H}^{n}$ ) is similar to that of the affine group " $a x+b$ " on $\partial \mathbf{U}$ (or $\mathbf{R}$ ).

In this paper we consider the unitary representation $U$ of $\mathbf{P}$ on $L^{2}\left(\mathbf{H}^{n}\right)$ defined by

$$
U_{(z, t, \rho)} f\left(z^{\prime}, t^{\prime}\right)=\rho^{-\frac{n+1}{2}} f\left(T_{(z, t, \rho)}^{-1}\left(z^{\prime}, t^{\prime}\right)\right)
$$




$$
\begin{array}{r}
=\rho^{-\frac{n+1}{2}} f\left(\frac{z^{\prime}-z}{\sqrt{\rho}}, \frac{t^{\prime}-t-2 \operatorname{Im} z \overline{z^{\prime}}}{\rho}\right), \\
f \in L^{2}\left(\mathbf{H}^{n}\right),(z, t, \rho) \in \mathbf{P} .
\end{array}
$$

$U$ is reducible on $L^{2}\left(\mathbf{H}^{n}\right)$. We shall decompose $L^{2}\left(\mathbf{H}^{n}\right)$ into the direct sum of the irreducible invariant closed subspaces under $U$. The restrictions of $U$ on these subspaces are square-integrable and we shall give the characterization of the admissible condition in terms of the Fourier transform. Then we can define the wavelet transform, which leads to isometric operators from the irreducible invariant closed subspaces of $L^{2}\left(\mathbf{H}^{n}\right)$ to $L^{2, \nu}\left(\mathbf{U}^{n+1}\right)$, the weighted $L^{2}$-spaces on $\mathbf{U}^{n+1}$. By selectting a series of orthogonal admissible wavelets, we get the direct sum decomposition of $L^{2, \nu}\left(\mathbf{U}^{n+1}\right)$ with the first component $A^{\nu}\left(\mathbf{U}^{n+1}\right)$, the (weighted) Bergman space.

\section{Direct sum decomposition of $L^{2}\left(\mathbf{H}^{n}\right)$.}

In order to decompose $L^{2}\left(\mathbf{H}^{n}\right)$, we state some facts of harmonic analysis on the Heisenberg group (cf. [3]). Let $\alpha=\left(\alpha_{1}, \cdots, \alpha_{n}\right) \in\left(\mathbf{Z}^{+}\right)^{n}$, where $\mathbf{Z}^{+}=$ $\{0,1,2, \cdots\}$. Set $\iota=(1, \cdots, 1) \in\left(\mathbf{Z}^{+}\right)^{n}, \iota_{j}=(0, \cdots, 1, \cdots, 0) \in\left(\mathbf{Z}^{+}\right)^{n}$ where the 1 in the $j$ th position. $(0, \cdots, 0) \in\left(\mathbf{Z}^{+}\right)^{n}$ will be written simply as 0 . Let $\mathcal{H}$ be a Hilbert space with fixed orthonormal basis $\left\{E_{\alpha}: \alpha \in\left(\mathbf{Z}^{+}\right)^{n}\right\}$. For $\lambda \in \mathbf{R} \backslash\{0\}$, we define the closed linear operators $W_{\lambda, j}$ and $W_{\lambda, j}^{+}$on $\mathcal{H}, j=1, \cdots, n$, in the following way:

$$
W_{\lambda, j} E_{\alpha}= \begin{cases}\left(2 \alpha_{j}|\lambda|\right)^{\frac{1}{2}} E_{\alpha-\iota_{j}}, & \text { if } \quad \lambda>0, \\ \left(2\left(\alpha_{j}+1\right)|\lambda|\right)^{\frac{1}{2}} E_{\alpha+\iota_{j}}, & \text { if } \quad \lambda<0,\end{cases}
$$

and

$$
W_{\lambda, j}^{+}=W_{-\lambda, j}
$$

where $E_{0-e_{j}}=0, j=1, \cdots, n$. Write $z W_{\lambda}=\sum_{j=1}^{n} z_{j} W_{\lambda, j}$ and $\bar{z} W_{\lambda}^{+}=$ $\sum_{j=1}^{n} \overline{z_{j}} W_{\lambda, j}^{+}$. Then $\pi_{\lambda}(z, t)=e^{-i \lambda t} \exp \left(-z W_{\lambda}+\bar{z} W_{\lambda}^{+}\right)$is an irreducible unitary representation of $\mathbf{H}^{n}$ on $\mathcal{H}$. The Fourier transform of a function $f \in L^{1}\left(\mathbf{H}^{n}\right)$ is the operator valued function defined by

$$
\widehat{f}(\lambda)=\int_{\mathbf{H}^{n}} f(z, t) \pi_{\lambda}(z, t) d z d t .
$$

We have the Plancherel formula

$$
\|f\|_{L^{2}\left(\mathbf{H}^{n}\right)}=\left\{\frac{2^{n-1}}{\pi^{n+1}} \int_{-\infty}^{+\infty}\|\widehat{f}(\lambda)\|_{H-S}^{2}|\lambda|^{n} d \lambda\right\}^{\frac{1}{2}}, \quad f \in L^{1}\left(\mathbf{H}^{n}\right) \cap L^{2}\left(\mathbf{H}^{n}\right),
$$


where $\|\cdot\|_{H-S}$ denotes the Hilbert-Schmidt norm of operators. The Plancherel formula is equivalent to

$$
\langle f, g\rangle_{L^{2}\left(\mathbf{H}^{n}\right)}=\frac{2^{n-1}}{\pi^{n+1}} \int_{-\infty}^{+\infty} \operatorname{tr}\left(\widehat{g}(\lambda)^{*} \widehat{f}(\lambda)\right)|\lambda|^{n} d \lambda, \quad f, g \in L^{1}\left(\mathbf{H}^{n}\right) \cap L^{2}\left(\mathbf{H}^{n}\right)
$$

which allows us to extend the Fourier transform to the tempered distributions on $\mathbf{H}^{n}$ by duality (cf. [4]). As a consequence of (2.3), we have the inversion of the Fourier transform

$$
f(z, t)=\frac{2^{n-1}}{\pi^{n+1}} \int_{-\infty}^{+\infty} \operatorname{tr}\left(\pi_{\lambda}(z, t)^{*} \widehat{f}(\lambda)\right)|\lambda|^{n} d \lambda
$$

provided that the integral converges. Suppose $\rho>0$. Let

$$
f_{\rho}(z, t)=\rho^{-(n+1)} f\left(\frac{z}{\sqrt{\rho}}, \frac{t}{\rho}\right) .
$$

Then

$$
\widehat{f}_{\rho}(\lambda)=\widehat{f}(\rho \lambda)
$$

Let $f * g$ be the convolution of $f$ and $g$, i.e.,

$$
f * g(z, t)=\int_{\mathbf{H}^{n}} f\left(z^{\prime}, t^{\prime}\right) g\left(\left(z^{\prime}, t^{\prime}\right)^{-1}(z, t)\right) d z d t
$$

Then

$$
\widehat{f * g}(\lambda)=\widehat{f}(\lambda) \widehat{g}(\lambda)
$$

We also note the following facts: If $\tilde{f}(z, t)=\overline{f\left((z, t)^{-1}\right)}=\overline{f(-z,-t)}$, then

$$
\widehat{\widetilde{f}}(\lambda)=\widehat{f}(\lambda)^{*}
$$

where $\widehat{f}(\lambda)^{*}$ denotes the adjoint of $\widehat{f}(\lambda)$, and if $g(z, t)=f(-\bar{z},-t)$, then

$$
\widehat{g}(\lambda)=\widehat{f}(-\lambda) \text {. }
$$

Let $L_{m}^{(\nu)}(s)$ be the Laguerre polynomials defined by

$$
L_{m}^{(\nu)}(s)=\sum_{\mu=0}^{m}\left(\begin{array}{l}
m+\nu \\
m-\mu
\end{array}\right) \frac{(-s)^{\mu}}{\mu !}
$$




$$
=\frac{1}{m !} e^{s} s^{-\nu}\left(\frac{d}{d s}\right)^{m}\left(e^{-s} s^{m+\nu}\right), \quad \nu>-1, m \in \mathbf{Z}^{+} .
$$

For $\alpha, \beta \in\left(\mathbf{Z}^{+}\right)^{n}, u \in \mathbf{R}^{n}$. We shall write

$$
L_{\alpha}^{(\beta)}(u)=\prod_{j=1}^{n} L_{\alpha_{j}}^{\left(\beta_{j}\right)}\left(u_{j}\right) .
$$

It is useful to calculate the matrix coefficients of the representation $\pi_{\lambda}(z, t)$ (cf. [6] and [16]). Let $\alpha, \beta \in\left(Z^{+}\right)^{n}, z \in \mathbf{C}^{n}$. Write $\alpha_{j} \vee \beta_{j}=\max \left(\alpha_{j}, \beta_{j}\right), \alpha_{j} \wedge$ $\beta_{j}=\min \left(\alpha_{j}, \beta_{j}\right),\left(\alpha_{j}-\beta_{j}\right)^{+}=\max \left(\alpha_{j}-\beta_{j}, 0\right),\left(\alpha_{j}-\beta_{j}\right)^{-}=\max \left(\beta_{j}-\right.$ $\left.\alpha_{j}, 0\right), j=1, \cdots, n$, and $\alpha \vee \beta=\left(\left(\alpha_{1} \vee \beta_{1}\right), \cdots,\left(\alpha_{n} \vee \beta_{n}\right)\right), \alpha \wedge \beta=\left(\left(\alpha_{1} \wedge\right.\right.$ $\left.\left.\beta_{1}\right), \cdots,\left(\alpha_{n} \wedge \beta_{n}\right)\right),(\alpha-\beta)^{+}=\left(\left(\alpha_{1}-\beta_{1}\right)^{+} \ldots,\left(\alpha_{n}-\beta_{n}\right)^{+}\right),(\alpha-\beta)^{-}=\left(\left(\alpha_{1}-\right.\right.$ $\left.\left.\beta_{1}\right)^{-}, \cdots,\left(\alpha_{n}-\beta_{n}\right)^{-}\right),|\alpha-\beta|=\sum_{j=1}^{n}\left|\alpha_{j}-\beta_{j}\right|,\|\alpha-\beta\|=\left(\left|\alpha_{1}-\beta_{1}\right|, \cdots, \mid \alpha_{n}-\right.$ $\left.\beta_{n} \mid\right), \alpha !=\alpha_{1} !, \cdots, \alpha_{n} !, z^{\alpha}=z_{1}^{\alpha_{1}} \cdots z_{n}^{\alpha_{n}},|z|^{2}=\sum_{j=1}^{n}\left|z_{j}\right|^{2},\|z\|^{2}=\left(\left|z_{1}\right|^{2}, \cdots\right.$, $\left.\left|z_{n}\right|^{2}\right)$. Then we have

$$
\begin{aligned}
\left\langle\pi_{\lambda}(z, t) E_{\alpha}, E_{\beta}\right\rangle_{\mathcal{H}}= & \left(\frac{(\alpha \wedge \beta) !}{(\alpha \vee \beta) !}\right)^{\frac{1}{2}} e^{-i \lambda t} e^{-\lambda|z|^{2}}(2 \lambda)^{\frac{|\alpha-\beta|}{2}} \\
& \cdot(-z)^{(\alpha-\beta)^{+}}(\bar{z})^{(\alpha-\beta)^{-}} L_{\alpha \wedge \beta}^{(\|\alpha-\beta\|)}\left(2 \lambda\|z\|^{2}\right), \quad \text { if } \quad \lambda>0,
\end{aligned}
$$

and

$$
\left\langle\pi_{\lambda}(z, t) E_{\alpha}, E_{\beta}\right\rangle_{\mathcal{H}}=\left\langle\pi_{-\lambda}(-\bar{z},-t) E_{\alpha}, E_{\beta}\right\rangle_{\mathcal{H}} \quad \text { if } \quad \lambda<0 .
$$

Now we are going to decompose $L^{2}\left(\mathbf{H}^{n}\right)$ into the direct sum of the irreducible invariant closed subspaces under the unitary representation $U$ of $\mathbf{P}$ defined by (1.11). In consideration of that $L^{2}(\mathbf{R})=H^{2}(\mathbf{R}) \oplus \bar{H}^{2}(\mathbf{R})$, one may give the decomposition of $L^{2}\left(\mathbf{H}^{n}\right)$ as $L^{2}\left(\mathbf{H}^{n}\right)=H^{+} \oplus H^{-}$, where

$$
\begin{array}{lll}
H^{+}=\left\{f \in L^{2}\left(\mathbf{H}^{n}\right):\right. & \widehat{f}(\lambda)=0 \quad \text { if } & \lambda<0\} \\
H^{-}=\left\{f \in L^{2}\left(\mathbf{H}^{n}\right):\right. & \widehat{f}(\lambda)=0 \quad \text { if } & \lambda>0\} .
\end{array}
$$

But $H^{+}$and $H^{-}$are reducible under $U$. Let $\mathcal{P}_{\alpha}$ denote the orthogonal projection from $\mathcal{H}$ to the one-dimensional subspace $\mathcal{H}_{\alpha}$ spanned by $E_{\alpha}$. Given $\alpha \in\left(\mathbf{Z}^{+}\right)^{n}, \sigma=+$ or - . We define the operator $P_{\alpha}^{\sigma}$ on $L^{2}\left(\mathbf{H}^{n}\right)$ in terms of Fourier transform by

$$
\widehat{P_{\alpha}^{+}} f(\lambda)= \begin{cases}\widehat{f}(\lambda) \mathcal{P}_{\alpha}, & \text { if } \lambda>0, \\ 0, & \text { if } \lambda<0,\end{cases}
$$




$$
\widehat{P_{\alpha}^{-}} f(\lambda)= \begin{cases}0, & \text { if } \quad \lambda>0 \\ \widehat{f}(\lambda) \mathcal{P}_{\alpha}, & \text { if } \quad \lambda<0 .\end{cases}
$$

So $P_{\alpha}^{\sigma}$ is an orthogonal projection. Let $H_{\alpha}^{\sigma}$ denote the range of $P_{\alpha}^{\sigma}$, which can be characterized in terms of the Fourier transform as following:

$$
\begin{aligned}
& H_{\alpha}^{+}=\left\{f \in L^{2}\left(\mathbf{H}^{n}\right): \widehat{f}(\lambda)=\widehat{f}(\lambda) \mathcal{P}_{\alpha} \quad \text { and } \quad \widehat{f}(\lambda)=0 \quad \text { if } \quad \lambda<0\right\}, \\
& H_{\alpha}^{-}=\left\{f \in L^{2}\left(\mathbf{H}^{n}\right): \widehat{f}(\lambda)=\widehat{f}(\lambda) \mathcal{P}_{\alpha} \quad \text { and } \quad \widehat{f}(\lambda)=0 \quad \text { if } \quad \lambda>0\right\} .
\end{aligned}
$$

We have the following theorem.

Theorem 1. Let $H_{\alpha}^{\sigma}$ be defined as above. Then $H_{\alpha}^{\sigma}$ is an irreducible invariant closed subspaces of $L^{2}\left(\mathbf{H}^{n}\right)$ under the unitary representation $U$ of $\mathbf{P}$ defined by (1.11), and we have the direct sum decomposition

$$
L^{2}\left(\mathbf{H}^{n}\right)=\bigoplus_{\alpha \in\left(\mathbf{Z}^{+}\right)^{n}}\left(H_{\alpha}^{+} \bigoplus H_{\alpha}^{-}\right)
$$

Proof. By (2.3), it is easy to see that the $H_{\alpha}^{\sigma}$ 's are mutually orthogonal closed subspaces of $L^{2}\left(\mathbf{H}^{n}\right)$ and $L^{2}\left(\mathbf{H}^{n}\right)$ is the direct sum of $H_{\alpha}^{\sigma}$ 's, i.e.,

$$
L^{2}\left(\mathbf{H}^{n}\right)=\bigoplus_{\alpha \in\left(\mathbf{Z}^{+}\right)^{n}}\left(H_{\alpha}^{+} \bigoplus H_{\alpha}^{-}\right) .
$$

All we need is to prove that the $H_{\alpha}^{\sigma}$ is an irreducible invariant subspace. Suppose $f \in L^{2}\left(\mathbf{H}^{n}\right)$. Because $\widehat{U_{(z, t, \rho)}} f(\lambda)=\rho^{\frac{n+1}{2}} \pi_{\lambda}(z, t) \widehat{f}(\rho \lambda)$, from (2.12) and (2.13), it is easy to see that $H_{\alpha}^{\sigma}$ is invariant under $U$. Let $V$ be a non-zero invariant closed subspace of $H_{\alpha}^{\sigma}$ under $U$ and $V^{\perp}$ the orthogonal complement of $V$ in $H_{\alpha}^{\sigma}$. Taking a function $g \in V$, not identically zero. Soppose $f \in V^{\perp}$, then $\left\langle f, U_{(z, t, \rho)} g\right\rangle_{L^{2}\left(\mathbf{H}^{n}\right)}=0$. Note that

$$
\left\langle f, U_{(z, t, \rho)} g\right\rangle_{L^{2}\left(\mathbf{H}^{n}\right)}=\rho^{\frac{n+1}{2}} f * \widetilde{g}_{\rho}(z, t) .
$$

By (2.5), (2.6) and (2.7), we have

$$
\int_{\mathbf{H}^{n}}\left\langle f, U_{(z, t, \rho)} g\right\rangle_{L^{2}\left(\mathbf{H}^{n}\right)} \pi_{\lambda}(z, t) d z d t=\rho^{\frac{n+1}{2}} \widehat{f}(\lambda) \widehat{\widetilde{g}}(\rho \lambda)=\rho^{\frac{n+1}{2}} \widehat{f}(\lambda) \widehat{g}(\rho \lambda)^{*} .
$$

Therefore,

$$
\rho^{\frac{n+1}{2}} \widehat{f}(\lambda) \widehat{g}(\rho \lambda)^{*}=0, \quad \text { for any } \quad \rho>0 .
$$


Because $\widehat{f}(\lambda)=\widehat{f}(\lambda) \mathcal{P}_{\alpha}, \widehat{g}(\rho \lambda)^{*}=\mathcal{P}_{\alpha} \widehat{g}(\rho \lambda)^{*}, \widehat{g}(\lambda) \not \equiv 0$, we have $\widehat{f}(\lambda) \equiv$ 0 . Hence $f$ is identically zero. This proves that $H_{\alpha}^{\sigma}$ is irreducible under $U$.

We shall give the explaination of $H_{\alpha}^{\sigma}$. $H_{0}^{+}$is exactly the Hardy space $H^{2}\left(\mathbf{H}^{n}\right)$, which is characterized in terms of Fourier transform by

$$
H^{2}\left(\mathbf{H}^{n}\right)=\left\{f \in L^{2}\left(\mathbf{H}^{n}\right): \widehat{f}(\lambda)=\widehat{f}(\lambda) \mathcal{P}_{0} \quad \text { and } \quad \widehat{f}(\lambda)=0 \quad \text { if } \quad \lambda<0\right\},
$$

where $\mathcal{P}_{0}$ is the orthogonal projection from $\mathcal{H}$ to $\mathcal{H}_{0}$ spanned by $E_{0}$. This characterization of $H^{2}\left(\mathbf{H}^{n}\right)$ was obtained by Ogden-Vági (cf. [12]). They called $\mathcal{H}_{0}$ the vacuum state. Set $Z_{j}=\frac{\partial}{\partial z_{j}}+i \overline{z_{j}} \frac{\partial}{\partial t}, \overline{Z_{j}}=\frac{\partial}{\partial \overline{z_{j}}}-i z_{j} \frac{\partial}{\partial t}, j=$ $1, \cdots, n . Z_{j}$ and $\overline{Z_{j}}$ are left-invariant differential operators on $\mathbf{H}^{n}$ and satisfy

$$
\left\{\begin{array}{l}
\widehat{Z_{j} f}(\lambda)=\widehat{f}(\lambda) W_{\lambda, j}, \\
\widehat{\widehat{Z_{j}}} f(\lambda)=-\widehat{f}(\lambda) W_{\lambda, j}^{+},
\end{array} \quad j=1, \cdots, n,\right.
$$

where $Z_{j} f$ and $\overline{Z_{j}} f$ are taken in the sense of distributions (see $\left.[\mathbf{3}]\right) . \overline{Z_{j}}, j=$ $1, \cdots, n$, can be explained as the restrictions of the tangential CauchyRiemann operators on $\partial \mathbf{U}^{n+1}=\mathbf{H}^{n}$ (cf. [14, Chapter 18]) and make up the $\bar{\partial}_{b}$ complex in essence (cf. [2]). The Hardy space $H^{2}\left(\mathbf{H}^{n}\right)$ can be characterized by

$$
H^{2}\left(\mathbf{H}^{n}\right)=\left\{f \in L^{2}\left(\mathbf{H}^{n}\right): \overline{Z_{j}} f=0 \quad \text { for } \quad j=1, \cdots, n\right\} .
$$

Now $H_{\alpha}^{+}$can be explained as following. Let $\bar{Z}^{\alpha}={\overline{Z_{1}}}^{\alpha_{1}} \cdots{\overline{Z_{n}}}^{\alpha_{n}}$. Set

$$
A_{\alpha}^{+}=\left\{f \in L^{2}\left(\mathbf{H}^{n}\right): \bar{Z}^{\alpha+\iota_{j}} f=0 \quad \text { for } \quad j=1, \cdots, n\right\} .
$$

Then

$$
A_{\alpha}^{+}=\bigoplus_{0 \leq \beta \leq \alpha} H_{\beta}^{+} .
$$

Similarly, $H_{0}^{-}$is the conjugate Hardy space $\overline{H^{2}}\left(\mathbf{H}^{n}\right)$, which is characterized by

$$
\overline{H^{2}}\left(\mathbf{H}^{n}\right)=\left\{f \in L^{2}\left(\mathbf{H}^{n}\right): Z_{j} f=0 \quad \text { for } \quad j=1, \cdots, n\right\} .
$$

Set

$$
A_{\alpha}^{-}=\left\{f \in L^{2}\left(\mathbf{H}^{n}\right): Z^{\alpha+\iota_{j}} f=0 \quad \text { for } \quad j=1, \cdots, n\right\} .
$$


Then

$$
A_{\alpha}^{-}=\bigoplus_{0 \leq \beta \leq \alpha} H_{\beta}^{-}
$$

As we have seen in Theorem $1, L^{2}\left(\mathbf{H}^{n}\right)$ has infinitely many irreducible invariant closed subspaces under $U$. This is different from $L^{2}(\mathbf{R})$, which has only two irreducible invariant closed subspaces $H^{2}(\mathbf{R})$ and $\overline{H^{2}}(\mathbf{R})$. But it will be seen in $\S 4$ that we may only consider the wavelat transform on spaces $H^{2}\left(\mathbf{H}^{n}\right)$ and $\overline{H^{2}}\left(\mathbf{H}^{n}\right)$, the difference between different indices $\alpha$ 's has no influences on the wavelet transform and the direct sum decomposition of the function spaces on $\mathbf{U}^{n+1}$.

\section{Admissible condition and wavelet transform.}

Given $\alpha \in\left(\mathbf{Z}^{+}\right)^{n}, \sigma=+$ or - . The restriction of $U$ on $H_{\alpha}^{\sigma}$ is squareintegrable in the sense of following: There exists in $H_{\alpha}^{\sigma}$ at least one function $\phi$, not identically zero, such that

$$
\frac{1}{\|\phi\|_{L^{2}\left(\mathbf{H}^{n}\right)}^{2}} \int_{\mathbf{P}}\left|\left\langle\phi, U_{(z, t, \rho)} \phi\right\rangle_{L^{2}\left(\mathbf{H}^{n}\right)}\right|^{2} \frac{d z d t d \rho}{\rho^{n+2}}=C_{\phi}<+\infty .
$$

(3.1) is called the admissible condition. If a function $\phi \in H_{\alpha}^{\sigma}$, not identically zero, satisfies the admissible condition (3.1), we say that $\phi$ is an admissible wavelet, and write $\phi \in A W_{\alpha}^{\sigma}$. Suppose $\phi \in A W_{\alpha}^{\sigma}$. We define the operator $W_{\phi}$ on $H_{\alpha}^{\sigma}$ by

$$
W_{\phi} f(z, t, \rho)=\left\langle f, U_{(z, t, \rho)} \phi\right\rangle_{L^{2}\left(\mathbf{H}^{n}\right)} .
$$

The function $W_{\phi} f(z, t, \rho)$ is called the (continuous) wavelet transform of $f$ with respect to $\phi$.

Before verifying the existence of admissible wavelets, we are going to give the characterization of the admissible condition like (1.3), which is not involved with the representation $U$. That is to say, the admissibility is an intrinsic property of a function. Let $\phi \in H_{\alpha}^{\sigma}$. By (2.14),

$$
\int_{\mathbf{H}^{n}}\left\langle\phi, U_{(z, t, \rho)} \phi\right\rangle_{L^{2}\left(\mathbf{H}^{n}\right)} \pi_{\lambda}(z, t) d z d t=\rho^{\frac{n+1}{2}} \widehat{\phi}(\lambda) \widehat{\phi}(\rho \lambda)^{*} .
$$

Using the Plancherel formula,

$$
\begin{aligned}
& \int_{\mathbf{P}}\left|\left\langle\phi, U_{(z, t, \rho)} \phi\right\rangle_{L^{2}\left(\mathbf{H}^{n}\right)}\right|^{2} \frac{d z d t d \rho}{\rho^{n+2}} \\
& =\frac{2^{n-1}}{\pi^{n+1}} \int_{0}^{+\infty}\left(\int_{-\infty}^{+\infty}\left\|\widehat{\phi}(\lambda) \widehat{\phi}(\rho \lambda)^{*}\right\|_{H-S}^{2}|\lambda|^{n} d \lambda\right) \frac{d \rho}{\rho}
\end{aligned}
$$




$$
=\frac{2^{n-1}}{\pi^{n+1}} \int_{0}^{+\infty}\left(\int_{-\infty}^{+\infty} \operatorname{tr}\left(\widehat{\phi}(\lambda)^{*} \widehat{\phi}(\lambda) \widehat{\phi}(\rho \lambda)^{*} \widehat{\phi}(\rho \lambda)\right)|\lambda|^{n} d \lambda\right) \frac{d \rho}{\rho} .
$$

Because $\phi \in H_{\alpha}^{\sigma}$, we have $\widehat{\phi}(\lambda)^{*} \widehat{\phi}(\lambda)=h(\lambda) \mathcal{P}_{\alpha}$ where $h(\lambda)=\|\widehat{\phi}(\lambda)\|_{H-S}^{2} \geq 0$, and $h(\lambda)=0$ if $\sigma=+, \lambda>0$ or $\sigma=-, \lambda<0$. Hence,

$$
\begin{aligned}
& \int_{\mathbf{P}}\left|\left\langle\phi, U_{(z, t, \rho)} \phi\right\rangle_{L^{2}\left(\mathbf{H}^{n}\right)}\right|^{2} \frac{d z d t d \rho}{\rho^{n+2}} \\
& =\frac{2^{n-1}}{\pi^{n+1}} \int_{0}^{+\infty}\left(\int_{-\infty}^{+\infty} h(\lambda) h(\rho \lambda)|\lambda|^{n} d \lambda\right) \frac{d \rho}{\rho} \\
& =\left(\int_{-\infty}^{+\infty} h\left(\lambda^{\prime}\right) \frac{d \lambda^{\prime}}{\left|\lambda^{\prime}\right|}\right)\left(\frac{2^{n-1}}{\pi^{n+1}} \int_{-\infty}^{+\infty} h(\lambda)|\lambda|^{n} d \lambda\right) \\
& =\left(\int_{-\infty}^{+\infty}\|\widehat{\phi}(\lambda)\|_{H-S}^{2} \frac{d \lambda}{|\lambda|}\right)\|\phi\|_{L^{2}\left(\mathbf{H}^{n}\right)}^{2} .
\end{aligned}
$$

We get the following theorem.

Theorem 2. Let $\phi \in H_{\alpha}^{\sigma}$, not identically zero. Then $\phi \in A W_{\alpha}^{\sigma}$ if and only if

$$
C_{\phi}=\int_{-\infty}^{+\infty}\|\widehat{\phi}(\lambda)\|_{H-S}^{2} \frac{d \lambda}{|\lambda|}<\infty
$$

Let $\phi, \psi \in A W_{\alpha}^{\sigma}$. We define the "inner product" of $\phi$ and $\psi$ on $A W_{\alpha}^{\sigma}$ by

$$
\langle\phi, \psi\rangle_{A W}=\int_{-\infty}^{+\infty} \operatorname{tr}\left(\widehat{\psi}(\lambda)^{*} \widehat{\phi}(\lambda)\right) \frac{d \lambda}{|\lambda|} .
$$

Theorem 3. Let $\phi, \psi \in A W_{\alpha}^{\sigma}, f, g \in H_{\alpha}^{\sigma}$. Then we have

$$
\left\langle W_{\psi} f, W_{\phi} g\right\rangle_{L^{2}\left(\mathbf{P}, d \mu_{l}\right)}=\langle\phi, \psi\rangle_{A W}\langle f, g\rangle_{L^{2}\left(\mathbf{H}^{n}\right)} .
$$

Proof. The proof of Theorem 3 is similar to the proof of Theorem 2. In fact,

$$
\begin{aligned}
& \left\langle W_{\psi} f, W_{\phi} g\right\rangle_{L^{2}\left(\mathbf{P}, d \mu_{l}\right)} \\
& =\int_{\mathbf{P}} W_{\psi} f(z, t, \rho) \overline{W_{\phi} g(z, t, \rho)} \frac{d z d t d \rho}{\rho^{n+2}} \\
& =\frac{2^{n-1}}{\pi^{n+1}} \int_{0}^{+\infty}\left(\int_{-\infty}^{+\infty} \operatorname{tr}\left(\widehat{g}(\lambda)^{*} \widehat{f}(\lambda) \widehat{\psi}(\rho \lambda)^{*} \widehat{\phi}(\rho \lambda)\right)|\lambda|^{n} d \lambda\right) \frac{d \rho}{\rho} \\
& =\left(\int_{-\infty}^{+\infty} \operatorname{tr}\left(\widehat{\psi}\left(\lambda^{\prime}\right)^{*} \widehat{\phi}\left(\lambda^{\prime}\right)\right) \frac{d \lambda^{\prime}}{\left|\lambda^{\prime}\right|}\right)\left(\frac{2^{n-1}}{\pi^{n+1}} \int_{-\infty}^{+\infty} \operatorname{tr}\left(\widehat{g}(\lambda)^{*} \widehat{f}(\lambda)\right)|\lambda|^{n} d \lambda\right) \\
& =\langle\phi, \psi\rangle_{A W}\langle f, g\rangle_{L^{2}\left(\mathbf{H}^{n}\right) .}
\end{aligned}
$$


Corollary. Let $\phi \in A W_{\alpha}^{\sigma}, f \in H_{\alpha}^{\sigma}$. Then

$$
\left\|W_{\phi} f\right\|_{L^{2}\left(\mathbf{P}, d \mu_{l}\right)}=C_{\phi}^{\frac{1}{2}}\|f\|_{L^{2}\left(\mathbf{H}^{n}\right)} .
$$

Another important consequence of Theorem 3 is the Calderón reproducing formula. We state it as a theorem.

Theorem 4. Let $\phi \in A W_{\alpha}^{\sigma}, f \in H_{\alpha}^{\sigma}$. Then

$$
f\left(z^{\prime}, t^{\prime}\right)=C_{\phi}^{-1} \int_{\mathbf{P}} W_{\phi} f(z, t, \rho), U_{(z, t, \rho)} \phi\left(z^{\prime}, t^{\prime}\right) \frac{d z d t d \rho}{\rho^{n+2}} .
$$

Proof. Let $\left\{g_{n}\right\}$ be a sequence in $C_{0}^{\infty}\left(\mathbf{H}^{n}\right)$ tending to the delta function supported at the point $\left(z^{\prime}, t^{\prime}\right)$ as $n \rightarrow \infty$. By Theorem 3,

$$
\left\langle f, g_{n}\right\rangle_{L^{2}\left(\mathbf{H}^{n}\right)}=\left\langle f, P_{\alpha}^{\sigma} g_{n}\right\rangle_{L^{2}\left(\mathbf{H}^{n}\right)}=C_{\phi}^{-1}\left\langle W_{\phi} f, W_{\phi} P_{\alpha}^{\sigma} g_{n}\right\rangle_{L^{2}\left(\mathbf{P}, d \mu_{l}\right)} .
$$

(3.7) is obtained by (3.8) and the limit argument.

We are interested in the question: How to get an admissible wavelet? Let $\mathcal{L}=-\frac{1}{2} \sum_{j=1}^{n}\left(Z_{j} \overline{Z_{j}}+\overline{Z_{j}} Z_{j}\right)$ be the "sub-Laplacian" on $\mathbf{H}^{n}$. By (2.21),

$$
\widehat{\mathcal{L} f}(\lambda)=\frac{1}{2} \widehat{f}(\lambda) \sum_{j=1}^{n}\left(W_{\lambda, j} W_{\lambda, j}^{+}+W_{\lambda, j}^{+} W_{\lambda, j}\right) .
$$

Hence,

$$
\widehat{\mathcal{L f}}(\lambda) \mathcal{P}_{\alpha}=(2|\alpha|+n)|\lambda| \widehat{f}(\lambda) \mathcal{P}_{\alpha} .
$$

Suppose $\mu>0$. Because $\mathcal{L}$ is a densely defined positive operator on $L^{2}\left(\mathbf{H}^{n}\right)$, the operator $\mathcal{L}^{\frac{\mu}{2}}$ is well-defined, and

$$
\widehat{\mathcal{L}^{\frac{\mu}{2}}} f(\lambda) \mathcal{P}_{\alpha}=((2|\alpha|+n)|\lambda|)^{\frac{\mu}{2}} \widehat{f}(\lambda) \mathcal{P}_{\alpha} .
$$

We call $\mathcal{L}^{\frac{\mu}{2}}$ the Riesz derivative of order $\mu$. Let $L_{\mu}^{2}\left(\mathbf{H}^{n}\right)=\left\{f \in L^{2}\left(\mathbf{H}^{n}\right)\right.$ : $\left.\mathcal{L}^{\frac{\mu}{2}} f \in L^{2}\left(\mathbf{H}^{n}\right)\right\}$. If $\mu$ is a positive integer, then $L_{\mu}^{2}\left(\mathbf{H}^{n}\right)$ is exactely the Sobolev space $S_{\mu}^{2}$ on $\mathbf{H}^{n}$ studied by Folland-Stein (cf. [2]). By Theorem 2, it is easy to obtain the following result: Let $f \in H_{\alpha}^{\sigma} \cap L_{n+1}^{2}\left(\mathbf{H}^{n}\right)$, not identically zero, then $\mathcal{L}^{\frac{n+1}{2}} f \in A W_{\alpha}^{\sigma}$. Another version of the result is following: Let $\phi \in H_{\alpha}^{\sigma}$, not identically zero, then $\phi \in A W_{\alpha}^{\sigma}$ if and only if $I_{n+1} \phi \in L^{2}\left(\mathbf{H}^{n}\right)$, where $I_{\mu}$ denotes the potential operator defined by $I_{\mu} f=\mathcal{L}^{-\frac{\mu}{2}} f$, i.e.,

$$
\widehat{I_{\mu} f}(\lambda) \mathcal{P}_{\alpha}=((2|\alpha|+n)|\lambda|)^{-\frac{\mu}{2}} \widehat{f}(\lambda) \mathcal{P}_{\alpha} .
$$


Now we give the examples of admissible wavelets.

Let $\alpha, \beta \in\left(\mathbf{Z}^{+}\right)^{n}, j \in \mathbf{Z}^{+}, \nu>-1$. We define the functions $\phi_{\beta, \alpha, j}^{\nu,+}$ and $\phi_{\beta, \alpha, j}^{\nu,-}$ in terms of the Fourier transform by

$$
\widehat{\phi}_{\beta, \alpha, j}^{\nu,+}(\lambda)= \begin{cases}\Gamma(\nu+1)^{-\frac{1}{2}}\left(\begin{array}{c}
\nu+j \\
j
\end{array}\right)^{-\frac{1}{2}}(2 \lambda)^{\frac{\nu+1}{2}} e^{-\lambda} L_{j}^{(\nu)}(2 \lambda) \mathcal{P}_{\beta, \alpha}, & \text { if } \lambda>0 \\
0, & \text { if } \lambda<0\end{cases}
$$

and

$$
\widehat{\phi}_{\beta, \alpha, j}^{\nu,-}(\lambda)=\widehat{\phi}_{\beta, \alpha, j}^{\nu,+}(-\lambda),
$$

where $\mathcal{P}_{\beta, \alpha}$ is the partial isometric operator on $\mathcal{H}$ defined by

$$
\mathcal{P}_{\beta, \alpha} E=\left\langle E, E_{\beta}\right\rangle_{\mathcal{H}} E_{\alpha}, \quad E \in \mathcal{H} .
$$

(We mention that $\mathcal{P}_{\alpha, \alpha}=\mathcal{P}_{\alpha}$.) The explicit expressions of $\phi_{\beta, \alpha, j}^{\nu,+}$ and $\phi_{\beta, \alpha, j}^{\nu,-}$ are given in the Appendix.

Given $\alpha, \beta \in\left(\mathbf{Z}^{+}\right)^{n}, j \in \mathbf{Z}^{+}, \nu>-1, \sigma=+$ or - . It is clear that $\phi_{\beta, \alpha, j}^{\nu, \sigma} \in H_{\beta}^{\sigma}$. From the orthogonal relations for the Laguerre polynimials (cf. [15, Chapter 5])

$\int_{0}^{+\infty} e^{-s} s^{\nu} L_{m}^{(\nu)}(s) L_{k}^{(\nu)}(s) d s=\Gamma(\nu+1)\left(\begin{array}{c}\nu+m \\ m\end{array}\right) \delta_{m k}, \quad \nu>-1, \quad m, k \in \mathbf{Z}^{+}$.

We have

$$
\begin{aligned}
& \int_{-\infty}^{+\infty}\left\|\widehat{\phi}_{\beta, \alpha, j}^{\nu, \sigma}(\lambda)\right\|_{H-S}^{2} \frac{d \lambda}{|\lambda|} \\
& =\Gamma(\nu+1)^{-1}\left(\begin{array}{c}
\nu+j \\
j
\end{array}\right)^{-1} \int_{0}^{+\infty} e^{-2 \lambda}(2 \lambda)^{\nu}\left(L_{j}^{(\nu)}(2 \lambda)\right)^{2} 2 d \lambda=1
\end{aligned}
$$

Therefore $\phi_{\beta, \alpha, j}^{\nu, \sigma}$ is an admissible wavelet. We also notice that $\left\{\phi_{\beta, \alpha, j}^{\nu, \sigma}: \alpha \in\right.$ $\left.\left(\mathbf{Z}^{+}\right)^{n}, j \in \mathbf{Z}^{+}\right\}$is an orthogonal set with respect to inner product $\langle,\rangle_{A W}$. In fact, if $\alpha \neq \alpha^{\prime}$, then

$$
\left\langle\phi_{\beta, \alpha, j}^{\nu, \sigma}, \phi_{\beta, \alpha^{\prime}, j^{\prime}}^{\nu, \sigma}\right\rangle_{A W}=\int_{-\infty}^{+\infty} \operatorname{tr}\left(\widehat{\phi}_{\beta, \alpha^{\prime}, j^{\prime}}^{\nu, \sigma}(\lambda)^{*} \widehat{\phi}_{\beta, \alpha, j}^{\nu, \sigma}(\lambda)\right) \frac{d \lambda}{|\lambda|}=0, \quad j, j^{\prime} \in \mathbf{Z}^{+} .
$$

If $j \neq j^{\prime}$. From the orthogonal relations for the Laguerre polynomials (3.15), we obtain

$$
\left\langle\phi_{\beta, \alpha, j}^{\nu, \sigma}, \phi_{\beta, \alpha, j^{\prime}}^{\nu, \sigma}\right\rangle_{A W}
$$




$$
\begin{aligned}
& =\int_{-\infty}^{+\infty} \operatorname{tr}\left(\widehat{\phi}_{\beta, \alpha, j^{\prime}}^{\nu, \sigma}(\lambda)^{*} \widehat{\phi}_{\beta, \alpha, j}^{\nu, \sigma}(\lambda)\right) \frac{d \lambda}{|\lambda|} \\
& =\Gamma(\nu+1)^{-1}\left(\begin{array}{c}
\nu+j \\
j
\end{array}\right)^{-1} \int_{0}^{+\infty} e^{-2 \lambda}(2 \lambda)^{\nu} L_{j}^{(\nu)}(2 \lambda) L_{j^{\prime}}^{(\nu)}(2 \lambda) 2 d \lambda \\
& =0, \quad \alpha \in\left(\mathbf{Z}^{+}\right)^{n} .
\end{aligned}
$$

By (3.16), (3.17) and (3.18), $\left\{\phi_{\beta, \alpha, j}^{\nu, \sigma}: \alpha \in\left(\mathbf{Z}^{+}\right)^{n}, j \in \mathbf{Z}^{+}\right\}$is an orthonormal set, i.e.,

$$
\left\langle\phi_{\beta, \alpha, j}^{\nu, \sigma}, \phi_{\beta, \alpha^{\prime}, j^{\prime}}^{\nu, \sigma}\right\rangle_{A W}=\delta_{\alpha, \alpha^{\prime}} \delta_{j, j^{\prime}}
$$

\section{Direct sum decomposition of $L^{2, \nu}\left(\mathbf{U}^{n+1}\right)$.}

Given $\alpha, \beta \in\left(\mathbf{Z}^{+}\right)^{n}, j \in \mathbf{Z}^{+}, \nu>-1, \sigma=+$ or - . Set

$$
A_{\beta, \alpha, j}^{\nu, \sigma}=\left\{W_{\phi_{\beta, \alpha, j}^{\nu, \sigma}} f: \quad f \in H_{\beta}^{\sigma}\right\} .
$$

By the Corollary of Theorem $3, A_{\beta, \alpha, j}^{\nu, \sigma}$ is a subspace of $L^{2}\left(\mathbf{P}, d \mu_{l}\right)$ and isomorphic with $H_{\beta}^{\sigma}$. A remarkable fact is that the difference between different indices $\beta$ 's makes no difference on spaces $A_{\beta, \alpha, j}^{\nu, \sigma}$. In other words, for any $\beta \in\left(\mathbf{Z}^{+}\right)^{n}$, we have

$$
A_{\beta, \alpha, j}^{\nu, \sigma}=A_{0, \alpha, j}^{\nu, \sigma}
$$

In fact, the map $T^{\beta}$ defined by

$$
\widehat{T^{\beta}} f(\lambda)=\widehat{f}(\lambda) \mathcal{P}_{0, \beta}, \quad f \in H_{\beta}^{\sigma},
$$

is the bijection from $H_{\beta}^{\sigma}$ onto $H_{0}^{\sigma}$. It is easy to verify that $W_{\phi_{\beta, \alpha, j}^{\nu, \sigma}} f=$ $W_{\phi_{0, \alpha, j}^{\nu, \sigma}}\left(T^{\beta} f\right)$. We shall write $A_{\alpha, j}^{\nu, \sigma}$ instead of $A_{0, \alpha, j}^{\nu, \sigma}$.

Theorem 5. Fix $\nu>-1$. Then

$$
L^{2}\left(\mathbf{P}, d \mu_{l}\right)=\bigoplus_{\substack{\alpha \in\left(\mathbf{Z}^{+}\right)^{n} \\ j \in \mathbf{Z}^{+}}}\left(A_{\alpha, j}^{\nu,+} \bigoplus A_{\alpha, j}^{\nu,-}\right) .
$$

Proof. From (2.3), it is easy to get

$$
A_{\alpha, j}^{\nu,+} \perp A_{\alpha^{\prime}, j^{\prime}}^{\nu,-}, \quad \alpha, \alpha^{\prime} \in\left(\mathbf{Z}^{+}\right)^{n}, \quad j, j^{\prime} \in \mathbf{Z}^{+} .
$$


It follows from Theorem 3 and (3.19) that

$$
A_{\alpha, j}^{\nu, \sigma} \perp A_{\alpha^{\prime}, j^{\prime}}^{\nu, \sigma}, \quad \text { if } \quad \alpha \neq \alpha^{\prime} \quad \text { or } \quad j \neq j^{\prime} .
$$

We see that the spaces $A_{\alpha, j}^{\nu, \sigma}$, are mutually orthogonal.

Let $F(z, t, \rho) \in L^{2}\left(\mathbf{P}, d \mu_{l}\right)$. Set $F_{\rho}(z, t)=F(z, t, \rho)$. For $\rho \in \mathbf{R}^{+}$almost every where, $F_{\rho}(z, t) \in L^{2}\left(\mathbf{H}^{n}\right)$. By the Plancherel formula, we have

$$
\begin{aligned}
& \|F\|_{L^{2}\left(\mathbf{P}, d \mu_{l}\right)} \\
& =\int_{0}^{+\infty}\left(\int_{\mathbf{H}^{n}}\left|F_{\rho}(z, t)\right|^{2} d z d t\right) \frac{d \rho}{\rho^{n+2}} \\
& =\frac{2^{n-1}}{\pi^{n+1}} \int_{0}^{+\infty}\left(\int_{-\infty}^{+\infty}\left\|\widehat{F}_{\rho}(\lambda)\right\|_{H-S}^{2}|\lambda|^{n} d \lambda\right) \frac{d \rho}{\rho^{n+2}} \\
& =\frac{2^{n-1}}{\pi^{n+1}} \int_{0}^{+\infty}\left(\int_{-\infty}^{+\infty} \sum_{\alpha, \beta \in\left(\mathbf{Z}^{+}\right)^{n}}\left|\left\langle\widehat{F}_{\rho}(\lambda) E_{\alpha}, E_{\beta}\right\rangle_{\mathcal{H}}\right|^{2}|\lambda|^{n} d \lambda\right) \frac{d \rho}{\rho^{n+2}} \\
& <+\infty .
\end{aligned}
$$

For $\alpha, \beta \in\left(\mathbf{Z}^{+}\right)^{n}, \lambda \in \mathbf{R} \backslash\{0\}$ almost everywhere, as the function of the variable $\rho, \rho^{-\frac{n+2}{2}}\left\langle\widehat{F}_{\rho}(\lambda) E_{\alpha}, E_{\beta}\right\rangle_{\mathcal{H}} \in L^{2}\left(\mathbf{R}^{+}\right)$. Set

$$
h_{j}(\rho)=\Gamma(\nu+1)^{-\frac{1}{2}}\left(\begin{array}{c}
\nu+j \\
j
\end{array}\right)^{-\frac{1}{2}}(2 \lambda)^{\frac{\nu+1}{2}} e^{-\lambda \rho} \rho^{\frac{\nu}{2}} L_{j}^{(\nu)}(2 \lambda \rho) .
$$

By the orthogonal relations and completeness of the Laguerre polynomials (cf. [15, Chapter 5]), $\left\{h_{j}(\rho): j \in \mathbf{Z}^{+}\right\}$is an orthonormal basis of $L^{2}\left(\mathbf{R}^{+}\right)$. Therefore

$$
\rho^{-\frac{n+2}{2}}\left\langle\widehat{F}_{\rho}(\lambda) E_{\alpha}, E_{\beta}\right\rangle_{\mathcal{H}}=\sum_{j=0}^{+\infty} a_{j}(\lambda, \alpha, \beta) h_{j}(\rho) .
$$

Equivalently,

$$
\rho^{-\frac{n+2}{2}} \widehat{F}_{\rho}(\lambda)=\sum_{\substack{\alpha, \beta \in\left(\mathbf{Z}^{+}\right)^{n} \\ j \in \mathbf{Z}^{+}}} a_{j}(\lambda, \alpha, \beta) h_{j}(\rho) \mathcal{P}_{\alpha, \beta} .
$$

We define the functions $f_{\alpha, j}^{+}$and $f_{\alpha, j}^{-}$in terms of the Fourier transform by

$$
\widehat{f}_{\alpha, j}^{+}(\lambda)= \begin{cases}\sum_{\beta \in\left(\mathbf{Z}^{+}\right)^{n}} a_{j}(\lambda, \alpha, \beta) \mathcal{P}_{0, \beta}, & \text { if } \lambda>0, \\ 0, & \text { if } \lambda<0,\end{cases}
$$


and

$$
\widehat{f}_{\alpha, j}^{-}(\lambda)=\widehat{f}_{\alpha, j}^{+}(-\lambda) .
$$

It is easy to verify that $f_{\alpha, j}^{\sigma} \in H_{0}^{\sigma}, \sigma=+$ or - . Moreover,

$$
\begin{aligned}
& \int_{\mathbf{H}^{n}} \sum_{\substack{\alpha \in\left(\mathbf{Z}^{+}\right)^{n} \\
j \in \mathbf{Z}^{+}}}\left(W_{\phi_{0, \alpha, j}^{\nu,+}} f_{\alpha, j}^{+}(z, t, \rho)+W_{\phi_{0, \alpha, j}^{\nu,-}} f_{\alpha, j}^{-}(z, t, \rho)\right) \pi_{\lambda}(z, t) d z d t \\
& =\rho^{\frac{n+1}{2}} \sum_{\substack{\alpha \in\left(\mathbf{Z}^{+}\right)^{n} \\
j \in \mathbf{Z}^{+}}}\left(\widehat{f}_{\alpha, j}^{+}(\lambda) \widehat{\phi}_{0, \alpha, j}^{\nu,+}(\rho \lambda)^{*}+\widehat{f}_{\alpha, j}^{-}(\lambda) \widehat{\phi}_{0, \alpha, j}^{\nu,-}(\rho \lambda)^{*}\right) \\
& =\rho^{\frac{n+2}{2}} \sum_{\substack{\alpha, \beta \in\left(\mathbf{Z}^{+}\right)^{n} \\
j \in \mathbf{Z}^{+}}} a_{j}(\lambda, \alpha, \beta) h_{j}(\rho) \mathcal{P}_{\alpha, \beta} \\
& =\widehat{F}_{\rho}(\lambda) .
\end{aligned}
$$

This implies

$$
F(z, t, \rho)=\sum_{\substack{\alpha \in\left(\mathbf{Z}^{+}\right)^{n} \\ j \in \mathbf{Z}^{+}}}\left(W_{\phi_{0, \alpha, j}^{\nu,+}} f_{\alpha, j}^{+}(z, t, \rho)+W_{\phi_{0, \alpha, j}^{\nu,-}} f_{\alpha, j}^{-}(z, t, \rho)\right) .
$$

The proof of Theorem 5 is completed.

Let $\nu \in \mathbf{R}$. We consider the weighted $L^{2}$-spaces $L^{2, \nu}\left(\mathbf{U}^{n+1}\right)$ defined by $(4.13)$

$$
L^{2, \nu}\left(\mathbf{U}^{n+1}\right)=\left\{F:\|F\|_{L^{2, \nu}\left(\mathbf{U}^{n+1}\right)}=\left(\int_{\mathbf{U}^{n+1}}|F(z, t, \rho)|^{2} \rho^{\nu} d z d t d \rho\right)^{\frac{1}{2}}<+\infty\right\} .
$$

Specifically $L^{2}\left(\mathbf{P}, d \mu_{l}\right)=L^{2,-(n+2)}\left(\mathbf{U}^{n+1}\right)$ under the identification of $\mathbf{P}$ with $\mathbf{U}^{n+1}$. If $\nu>-1$, there exist holomorphic functions in $L^{2, \nu}\left(\mathbf{U}^{n+1}\right)$. The (weighted) Bergman space $A^{\nu}\left(\mathbf{U}^{n+1}\right)$ is the subspace of all holomorphic functions in $L^{2, \nu}\left(\mathbf{U}^{n+1}\right)$, i.e.,

$$
A^{\nu}\left(\mathbf{U}^{n+1}\right)=\left\{F \in L^{2, \nu}\left(\mathbf{U}^{n+1}\right): F \text { is holomorphic on } \mathbf{U}^{n+1}\right\}, \quad \nu>-1 \text {. }
$$

Given $\alpha, \beta \in\left(\mathbf{Z}^{+}\right)^{n}, j \in \mathbf{Z}^{+}, \nu \in \mathbf{R}, \nu^{\prime}>-1, \sigma=+$ or - . Set

$$
A_{\beta, \alpha, j}^{\nu, \nu^{\prime}, \sigma}=\left\{\rho^{-\frac{\nu+n+2}{2}} W_{\phi_{\beta, \alpha, j}^{\nu^{\prime}, \sigma}} f: \quad f \in H_{\beta}^{\sigma}\right\}
$$


Especially, $A_{\beta, \alpha, j}^{-(n+2), \nu^{\prime}, \sigma}=A_{\beta, \alpha, j}^{\nu^{\prime}, \sigma}$. It is clear that $A_{\beta, \alpha, j}^{\nu, \nu^{\prime}, \sigma}$ is a subspace of $L^{2, \nu}\left(\mathbf{U}^{n+1}\right)$ and isomorphic with $H_{\beta}^{\sigma}$. Similar to (4.2), we have

$$
A_{\beta, \alpha, j}^{\nu, \nu^{\prime}, \sigma}=A_{0, \alpha, j}^{\nu, \nu^{\prime}, \sigma} \quad \text { for any } \quad \beta \in\left(\mathbf{Z}^{+}\right)^{n} .
$$

We shall write $A_{\alpha, j}^{\nu, \nu^{\prime}, \sigma}$ instead of $A_{0, \alpha, j}^{\nu, \nu^{\prime}, \sigma}$. Also we have

Theorem 6. Let $\nu \in \mathbf{R}$. Fix $\nu^{\prime}>-1$. Then

$$
L^{2, \nu}\left(\mathbf{U}^{n+1}\right)=\bigoplus_{\substack{\alpha \in\left(\mathbf{Z}^{+}\right)^{n} \\ j \in \mathbf{Z}^{+}}}\left(A_{\alpha, j}^{\nu, \nu^{\prime},+} \bigoplus A_{\alpha, j}^{\nu, \nu^{\prime},-}\right) .
$$

The proof of Theorem 6 is similar to the proof of Theorem 5 and we omit it.

Let $K_{\alpha, j}^{\nu, \nu^{\prime}, \sigma}$ denotes the reproducing kernel of $A_{\alpha, j}^{\nu, \nu^{\prime}, \sigma}$. It is easy to see that

$$
\begin{aligned}
& K_{\alpha, j}^{\nu, \nu^{\prime}, \sigma}\left(\left(z^{\prime}, t^{\prime}, \rho^{\prime}\right),(z, t, \rho)\right) \\
& =\left(\rho \rho^{\prime}\right)^{-\frac{\nu+n+2}{2}} \int_{\mathbf{H}^{n}} U_{\left(z^{\prime}, t^{\prime}, \rho^{\prime}\right)} \phi_{0, \alpha, j}^{\nu^{\prime}, \sigma}(w, s) \overline{U_{(z, t, \rho)} \phi_{0, \alpha, j}^{\nu^{\prime}, \sigma}(w, s)} d w d s .
\end{aligned}
$$

The explicit expressions of $K_{\alpha, j}^{\nu, \nu^{\prime}, \sigma}$ are given in the Appendix.

In practice we are interested in the cases $\nu=\nu^{\prime}>-1$. The reproducing kernel of $A_{0,0}^{\nu, \nu,+}$ reads of

$$
\begin{aligned}
& K_{0,0}^{\nu, \nu,+}\left(\left(z^{\prime}, t^{\prime}, \rho^{\prime}\right),(z, t, \rho)\right) \\
& =\frac{1}{4 \pi^{n+1}} \frac{\Gamma(\nu+n+2)}{\Gamma(\nu+1)}\left(\frac{2}{\left|z-z^{\prime}\right|^{2}+\rho+\rho^{\prime}-i\left(t-t^{\prime}+2 \operatorname{Im} z \overline{z^{\prime}}\right)}\right)^{\nu+n+2},
\end{aligned}
$$

which is just the (weighted) Bergman kernel, the reproducing kernel of $A^{\nu}\left(\mathbf{U}^{n+1}\right)$ (cf. [11]). Therefore $A_{0,0}^{\nu, \nu,+}$ is exactly the (weighted) Bergman space $A^{\nu}\left(\mathbf{U}^{n+1}\right)$.

It is not difficult to give the orthonormal bases of $A_{\alpha, j}^{\nu, \nu, \sigma}$. Let $\beta \in\left(\mathbf{Z}^{+}\right)^{n}$, $k \in \mathbf{Z}^{+}$. We define the functions $f_{\beta, k}^{\mu,+}$ and $f_{\beta, k}^{\mu,-}$ in terms of Fourier transform by

$$
\widehat{f}_{\beta, k}^{\mu,+}(\lambda)= \begin{cases}2 \pi^{\frac{n+1}{2}} \Gamma(\mu+1)^{-\frac{1}{2}}\left(\begin{array}{c}
\mu+k \\
k
\end{array}\right)^{-\frac{1}{2}}(2 \lambda)^{\frac{\mu-n}{2}} e^{-\lambda} L_{k}^{(\mu)}(2 \lambda) \mathcal{P}_{0, \beta}, & \text { if } \lambda>0 \\
0, & \text { if } \lambda<0\end{cases}
$$


and

$$
\widehat{f}_{\beta, k}^{\mu,-}(\lambda)=\widehat{f}_{\beta, k}^{\mu,+}(-\lambda),
$$

where $\mu$ may depend on $\beta$ but not on $k$ such that $\mu>-1$. It follows from the orthogonal relations and completeness of the Laguerre polynomials that $\left\{f_{\beta, k}^{\mu, \sigma}: \beta \in\left(\mathbf{Z}^{+}\right)^{n}, k \in \mathbf{Z}^{+}\right\}$is an orthonormal basis of $H_{0}^{\sigma}$. Let

$$
\psi_{\alpha, j, \beta, k}^{\nu, \mu, \sigma}(z, t, \rho)=\rho^{-\frac{\nu+n+2}{2}} W_{\phi_{0, \alpha, j}^{\nu, \sigma}} f_{\beta, k}^{\mu, \sigma}(z, t, \rho) .
$$

Then $\left\{\psi_{\alpha, j, \beta, k}^{\nu, \mu, \sigma}: \beta \in\left(\mathbf{Z}^{+}\right)^{n}, k \in \mathbf{Z}^{+}\right\}$is an orthonormal basis of $A_{\alpha, j}^{\nu, \nu, \sigma}$. The explicit expressions of $\psi_{\alpha, j, \beta, k}^{\nu, \mu, \sigma}$ are given in the Appendix. Especially, for $\alpha=0, j=0$, take $\mu=\nu+n+1+|\beta|$, we have

$$
\begin{aligned}
& e_{\beta, k}^{\nu}(z, t, \rho) \\
& =\psi_{0,0, \beta, k}^{\nu, \nu+n+1+|\beta|,+}(z, t, \rho) \\
& =\frac{1}{2 \pi^{\frac{n+1}{2}}}\left(\frac{\Gamma(\nu+n+2+|\beta|+k)}{\Gamma(\nu+1) \beta ! k !}\right)^{\frac{1}{2}} \frac{2^{\nu+n+2+|\beta|} z^{\beta}\left(|z|^{2}+\rho-1-i t\right)^{k}}{\left(|z|^{2}+\rho+1-i t\right)^{\nu+n+2+|\beta|+k}} .
\end{aligned}
$$

$\left\{e_{\beta, k}^{\nu}: \beta \in\left(\mathbf{Z}^{+}\right)^{n}, k \in \mathbf{Z}^{+}\right\}$is an orthonormal basis of $A^{\nu}\left(\mathbf{U}^{n+1}\right)$.

Let $L^{2, \nu}\left(\mathbf{B}^{n+1}\right)$ denote the weighted $L^{2}$-space defined by

$$
\begin{aligned}
& L^{2, \nu}\left(\mathbf{B}^{n+1}\right) \\
& =\left\{F(w):\|F\|_{L^{2, \nu}\left(\mathbf{B}^{n+1}\right)}=\left(\int_{\mathbf{B}^{n+1}}|F(w)|^{2}\left(1-|w|^{2}\right)^{\nu} d w\right)^{\frac{1}{2}}<+\infty\right\},
\end{aligned}
$$

where $w=\left(w_{1}, \cdots, w_{n}, w_{n+1}\right) \in \mathbf{B}^{n+1},|w|^{2}=\sum_{j=1}^{n+1}\left|w_{j}\right|^{2}, d w$ denotes the Lebesgue measure of $\mathbf{C}^{n+1}$. For $\nu>-1$, let $A^{\nu}\left(\mathbf{B}^{n+1}\right)$ denote the (weighted) Bergman space on $\mathbf{B}^{n+1}$, the subspace of all holomorphic functions in $L^{2, \nu}\left(\mathbf{B}^{n+1}\right)$. The Cayley transform leads to the isomorphic map from $A^{\nu}\left(\mathbf{U}^{n+1}\right)$ (and $\left.L^{2, \nu}\left(\mathbf{U}^{n+1}\right)\right)$ to $A^{\nu}\left(\mathbf{B}^{n+1}\right)$ (and $\left.L^{2, \nu}\left(\mathbf{B}^{n+1}\right)\right)$. Let $T$ denote the isomorphic map from $A^{\nu}\left(\mathbf{U}^{n+1}\right)$ to $A^{\nu}\left(\mathbf{B}^{n+1}\right)$ defined by

$$
T: f\left(z, z_{n+1}\right) \longmapsto F(w)=2 f(\Gamma(w))\left(1-w_{n+1}\right)^{-(\nu+n+2)},
$$

where $\Gamma$ is the Cayley transform defined by (1.7). Then $T^{-1}$ is given by

$$
T^{-1}: F(w) \longmapsto f\left(z, z_{n+1}\right)=\frac{1}{2} F\left(\Gamma^{-1}\left(z, z_{n+1}\right)\right)\left(\frac{2 i}{z_{n+1}+i}\right)^{\nu+n+2} .
$$


It is easy to get

$$
T e_{\beta, k}^{\nu}(w)=\frac{i^{|\beta|}}{\pi^{\frac{n+1}{2}}}\left(\frac{\Gamma(\nu+n+2+|\beta|+k)}{\Gamma(\nu+1) \beta ! k !}\right)^{\frac{1}{2}} w^{(\beta, k)}
$$

where $w^{(\beta, k)}=w_{1}^{\beta_{1}} \cdots w_{n}^{\beta_{n}} w_{n+1}^{k}$. We see that $\left\{T e_{\beta, k}^{\nu}:(\beta, k) \in\left(\mathbf{Z}^{+}\right)^{n+1}\right\}$ is just the orthonormal basis of $A^{\nu}\left(\mathbf{B}^{n+1}\right)$ which consists of the monomials of $w$.

\section{Appendix.}

In this appendix we give the explicit expressions of $\phi_{\beta, \alpha, j}^{\nu, \sigma}, K_{\alpha, j}^{\nu, \nu^{\prime}, \sigma}$ and $\psi_{\alpha, j, \beta, k}^{\nu, \mu, \sigma}$.

For $a \in \mathbf{C}, b, c \in \mathbf{C}^{k}$, let

$$
F_{2}(a ; b ; c ; w)=\sum_{m \in\left(\mathbf{Z}^{+}\right)^{k}} \frac{(a)_{|m|}\left(b_{1}\right)_{m_{1}} \cdots\left(b_{k}\right)_{m_{k}}}{\left(c_{1}\right)_{m_{1}} \cdots\left(c_{k}\right)_{m_{k}}} \frac{w^{m}}{m !}, \quad w \in \mathbf{C}^{k},
$$

be the hypergeometric function of several variables (cf. [1, Chapter 5]).

From the inversion of the Fourier transform and (2.10), we obtain

$$
\begin{aligned}
& \text { (A.1) } \\
& \phi_{\beta, \alpha, j}^{\nu,+}(z, t) \\
& =\frac{2^{n-1}}{\pi^{n+1}} \int_{-\infty}^{+\infty} \operatorname{tr}\left(\pi_{\lambda}(z, t)^{*} \widehat{\phi}_{\beta, \alpha, j}^{\nu,+}(\lambda)\right)|\lambda|^{n} d \lambda \\
& =\frac{2^{n-1}}{\pi^{n+1}} \Gamma(\nu+1)^{-\frac{1}{2}}\left(\begin{array}{c}
\nu+j \\
j
\end{array}\right)^{-\frac{1}{2}} \\
& \cdot \int_{0}^{+\infty}(2 \lambda)^{\frac{\nu+1}{2}} e^{-\lambda} L_{j}^{(\nu)}(2 \lambda)\left\langle\pi_{\lambda}(-z,-t) E_{\alpha}, E_{\beta}\right\rangle_{\mathcal{H}} \lambda^{n} d \lambda \\
& =\frac{1}{4 \pi^{n+1}}\left(\frac{(\alpha \wedge \beta) !}{(\alpha \vee \beta) !}\right)^{\frac{1}{2}} \Gamma(\nu+1)^{-\frac{1}{2}}\left(\begin{array}{c}
\nu+j \\
j
\end{array}\right)^{-\frac{1}{2}} z^{(\alpha-\beta)^{+}}(-\bar{z})^{(\alpha-\beta)^{-}} \\
& \cdot \sum_{\substack{0 \leq m \leq j \\
0 \leq \gamma \leq(\alpha \wedge \beta)}}\left(\begin{array}{c}
\nu+j \\
j-m
\end{array}\right) \frac{(\alpha \vee \beta) !}{((\alpha \wedge \beta)-\gamma) !(\|\alpha-\beta\|+\gamma) !} \frac{(-1)^{m+|\gamma|}\left(\|z\|^{2}\right)^{\gamma}}{m ! \gamma !} \\
& \cdot \Gamma\left(\frac{\nu+1+|\alpha-\beta|}{2}+n+1+m+|\gamma|\right) \\
& \cdot\left(\frac{2}{|z|^{2}+1-i t}\right)^{\frac{\nu+1+|\alpha-\beta|}{2}+n+1+m+|\gamma|} \\
& =\frac{1}{4 \pi^{n+1}} \frac{\Gamma\left(\frac{\nu+1+|\alpha-\beta|}{2}+n+1\right)}{(\|\alpha-\beta\|) ! \Gamma(\nu+1)}\left(\frac{(\alpha \vee \beta) ! \Gamma(\nu+j+1)}{(\alpha \wedge \beta) ! j !}\right)^{\frac{1}{2}} z^{(\alpha-\beta)^{+}}(-\bar{z})^{(\alpha-\beta)^{-}}
\end{aligned}
$$




$$
\begin{aligned}
& \cdot\left(\frac{2}{|z|^{2}+1-i t}\right) \stackrel{\frac{\nu+1+|\alpha-\beta|}{2}+n+1}{F_{2}}\left(\frac{\nu+1+|\alpha-\beta|}{2}+n+1 ;\right. \\
& \left.(-j,-(\alpha \wedge \beta)) ;(\nu+1,\|\alpha-\beta\|+\iota) ; \frac{2}{|z|^{2}+1-i t}\left(1,\|z\|^{2}\right)\right),
\end{aligned}
$$

where $-\frac{\pi}{2}<\arg \left(|z|^{2}+1-i t\right)<\frac{\pi}{2}$, and by (2.8), we have

$$
\phi_{\beta, \alpha, j}^{\nu,-}(z, t)=\phi_{\beta, \alpha, j}^{\nu,+}(-\bar{z},-t) .
$$

$$
\begin{aligned}
& K_{\alpha, j}^{\nu, \nu^{\prime},+}\left(\left(z^{\prime}, t^{\prime}, \rho^{\prime}\right),(z, t, \rho)\right) \\
& =\left(\rho \rho^{\prime}\right)^{-\frac{\nu+n+2}{2}} \int_{\mathbf{H}^{n}} U_{\left(z^{\prime}, t^{\prime}, \rho^{\prime}\right)} \phi_{0, \alpha, j}^{\nu^{\prime},+}(w, s) \overline{U_{(z, t, \rho)} \phi_{0, \alpha, j}^{\nu^{\prime},+}(w, s)} d w d s \\
& =\frac{2^{n-1}}{\pi^{n+1}}\left(\rho \rho^{\prime}\right)^{-\frac{\nu+1}{2}} \int_{-\infty}^{+\infty} \operatorname{tr}\left(\widehat{\phi}_{0, \alpha, j}^{\nu^{\prime},+}(\rho \lambda)^{*} \pi_{\lambda}(z, t)^{*} \pi_{\lambda}\left(z^{\prime}, t^{\prime}\right) \widehat{\phi}_{0, \alpha, j}^{\nu^{\prime},+}\left(\rho^{\prime} \lambda\right)\right)|\lambda|^{n} d \lambda \\
& =\frac{2^{n-1}}{\pi^{n+1}}\left(\rho \rho^{\prime}\right)^{-\frac{\nu+1}{2}} \\
& \cdot \int_{-\infty}^{+\infty} \operatorname{tr}\left(\pi_{\lambda}\left(z^{\prime}-z, t^{\prime}-t-2 \operatorname{Im} z \overline{z^{\prime}}\right) \widehat{\phi}_{0, \alpha, j}^{\nu^{\prime},+}\left(\rho^{\prime} \lambda\right) \widehat{\phi}_{0, \alpha, j}^{\nu^{\prime},+}(\rho \lambda)^{*}\right)|\lambda|^{n} d \lambda \\
& =\frac{2^{\nu^{\prime}+n}}{\pi^{n+1}} \Gamma\left(\nu^{\prime}+1\right)^{-1}\left(\begin{array}{c}
\nu^{\prime}+j \\
j
\end{array}\right)^{-1}\left(\rho \rho^{\prime}\right)^{\frac{\nu^{\prime}-\nu}{2}} \int_{0}^{+\infty} \lambda^{\nu^{\prime}+n+1} e^{-\left(\rho+\rho^{\prime}\right) \lambda} \\
& \cdot L_{j}^{\left(\nu^{\prime}\right)}(2 \rho \lambda) L_{j}^{\left(\nu^{\prime}\right)}\left(2 \rho^{\prime} \lambda\right)\left\langle\pi_{\lambda}\left(z^{\prime}-z, t^{\prime}-t-2 \operatorname{Im} z \overline{z^{\prime}}\right) E_{\alpha}, E_{\alpha}\right\rangle_{\mathcal{H}} d \lambda \\
& =\frac{1}{4 \pi^{n+1}} \Gamma\left(\nu^{\prime}+1\right)^{-1}\left(\begin{array}{c}
\nu^{\prime}+j \\
j
\end{array}\right)^{-1}\left(\rho \rho^{\prime}\right)^{\frac{\nu^{\prime}-\nu}{2}} \\
& \sum_{\substack{0 \leq m, k \leq j \\
0 \leq \gamma \leq \alpha}}\left(\begin{array}{c}
\nu^{\prime}+j \\
j-m
\end{array}\right)\left(\begin{array}{c}
\nu^{\prime}+j \\
j-k
\end{array}\right) \frac{\alpha !}{(\alpha-\gamma) ! \gamma !} \\
& \frac{(-1)^{m+k+|\gamma|} \rho^{m} \rho^{\prime k}\left(\left\|z-z^{\prime}\right\|^{2}\right)^{\gamma}}{m ! k ! \gamma !} \Gamma\left(\nu^{\prime}+n+2+m+k+|\gamma|\right) \\
& \cdot\left(\frac{2}{\left|z-z^{\prime}\right|^{2}+\rho+\rho^{\prime}-i\left(t-t^{\prime}+2 \operatorname{Im} z \overline{z^{\prime}}\right)}\right)^{\nu^{\prime}+n+2+m+k+|\gamma|} \\
& =\frac{1}{4 \pi^{n+1}} \frac{\Gamma\left(\nu^{\prime}+n+2\right) \Gamma\left(\nu^{\prime}+j+1\right)}{\Gamma\left(\nu^{\prime}+1\right)^{2} j !}\left(\rho \rho^{\prime}\right)^{\frac{\nu^{\prime}-\nu}{2}} \\
& \cdot\left(\frac{2}{\left|z-z^{\prime}\right|^{2}+\rho+\rho^{\prime}-i\left(t-t^{\prime}+2 \operatorname{Im} z \overline{z^{\prime}}\right)}\right)^{\nu^{\prime}+n+2} \\
& \cdot F_{2}\left(\nu^{\prime}+n+2 ;(-j,-j,-\alpha) ;\left(\nu^{\prime}+1, \nu^{\prime}+1, \iota\right) ;\right.
\end{aligned}
$$




$$
\left.\frac{2}{\left|z-z^{\prime}\right|^{2}+\rho+\rho^{\prime}-i\left(t-t^{\prime}+2 \operatorname{Im} z \overline{z^{\prime}}\right)}\left(\rho, \rho^{\prime},\left\|z-z^{\prime}\right\|^{2}\right)\right)
$$

where $-\frac{\pi}{2}<\arg \left(\left|z-z^{\prime}\right|^{2}+\rho+\rho^{\prime}-i\left(t-t^{\prime}+2 \operatorname{Im} z \overline{z^{\prime}}\right)\right)<\frac{\pi}{2}$, and

$$
K_{\alpha, j}^{\nu, \nu^{\prime},-}\left(\left(z^{\prime}, t^{\prime}, \rho^{\prime}\right),(z, t, \rho)\right)=K_{\alpha, j}^{\nu, \nu^{\prime},+}\left(\left(-\overline{z^{\prime}},-t^{\prime}, \rho^{\prime}\right),(-\bar{z},-t, \rho)\right) .
$$

$$
\begin{aligned}
& \psi_{\alpha, j, \beta, k}^{\nu, \mu,+}(z, t, \rho) \\
& =\frac{2^{n-1}}{\pi^{n+1}} \int_{-\infty}^{+\infty} \rho^{-\frac{\nu+1}{2}} \operatorname{tr}\left(\pi_{\lambda}(z, t)^{*} \widehat{f}_{\beta, k}^{\mu,+}(\lambda) \widehat{\phi}_{0, \alpha, j}^{\nu,+}(\rho \lambda)^{*}\right)|\lambda|^{n} d \lambda \\
& =\frac{2^{n}}{\pi^{\frac{n+1}{2}}} \Gamma(\mu+1)^{-\frac{1}{2}} \Gamma(\nu+1)^{-\frac{1}{2}}\left(\begin{array}{c}
\mu+k \\
k
\end{array}\right)^{-\frac{1}{2}}\left(\begin{array}{c}
\nu+j \\
j
\end{array}\right)^{-\frac{1}{2}} \\
& \cdot \int_{0}^{+\infty}(2 \lambda)^{\frac{\mu+\nu-n+1}{2}} e^{-(1+\rho) \lambda} L_{k}^{(\mu)}(2 \lambda) L_{j}^{(\nu)}(2 \rho \lambda)\left\langle\pi_{\lambda}(-z,-t) E_{\beta}, E_{\alpha}\right\rangle_{\mathcal{H}} \lambda^{n} d \lambda \\
& =\frac{1}{2 \pi^{\frac{n+1}{2}}}\left(\frac{(\alpha \wedge \beta) !}{(\alpha \vee \beta) !}\right)^{\frac{1}{2}} \Gamma(\mu+1)^{-\frac{1}{2}} \Gamma(\nu+1)^{-\frac{1}{2}} \\
& \cdot\left(\begin{array}{c}
\mu+k \\
k
\end{array}\right)^{-\frac{1}{2}}\left(\begin{array}{c}
\nu+j \\
j
\end{array}\right)^{-\frac{1}{2}} z^{(\alpha-\beta)^{-}}(-\bar{z})^{(\alpha-\beta)^{+}} \\
& \cdot \sum_{\substack{0 \leq l \leq k \\
0 \leq m \leq j \\
0 \leq \gamma \leq(\alpha \wedge \beta)}}\left(\begin{array}{c}
\mu+k \\
k-l
\end{array}\right)\left(\begin{array}{c}
\nu+j \\
j-m
\end{array}\right) \frac{(\alpha \vee \beta) !}{((\alpha \wedge \beta)-\gamma) !(\|\alpha-\beta\|+\gamma) !} \\
& \cdot \frac{(-1)^{l+m+|\gamma|} \rho^{m}\left(\|z\|^{2}\right)^{\gamma}}{l ! m ! \gamma !} \Gamma\left(\frac{\mu+\nu+n+1+|\alpha-\beta|}{2}+1+l+m+|\gamma|\right) \\
& \cdot\left(\frac{2}{|z|^{2}+\rho+1-i t}\right)^{\frac{\mu+\nu+n+1+|\alpha-\beta|}{2}+1+l+m+|\gamma|} \\
& =\frac{1}{2 \pi^{\frac{n+1}{2}}} \frac{\Gamma\left(\frac{\mu+\nu+n+1+|\alpha-\beta|}{2}+1\right)}{(\|\alpha-\beta\|) ! \Gamma(\mu+1) \Gamma(\nu+1)}\left(\frac{(\alpha \vee \beta) ! \Gamma(\mu+k+1) \Gamma(\nu+j+1)}{(\alpha \wedge \beta) ! k ! j !}\right)^{\frac{1}{2}} \\
& \cdot z^{(\alpha-\beta)^{-}}(-\bar{z})^{(\alpha-\beta)^{+}}\left(\frac{2}{|z|^{2}+\rho+1-i t}\right)^{\frac{\mu+\nu+n+1+|\alpha-\beta|}{2}+1} \\
& \cdot F_{2}\left(\frac{\mu+\nu+n+1+|\alpha-\beta|}{2}+1 ;(-k,-j,-(\alpha \wedge \beta))\right. \text {; } \\
& \left.(\mu+1, \nu+1,\|\alpha-\beta\|+\iota) ; \frac{2}{|z|^{2}+\rho+1-i t}\left(1, \rho,\|z\|^{2}\right)\right),
\end{aligned}
$$


where $-\frac{\pi}{2}<\arg \left(|z|^{2}+\rho+1-i t\right)<\frac{\pi}{2}$, and

$$
\psi_{\alpha, j, \beta, k}^{\nu, \mu,-}(z, t, \rho)=\psi_{\alpha, j, \beta, k}^{\nu, \mu,+}(-\bar{z},-t, \rho) .
$$

\section{References}

[1] A. Erdély et al, Higher transcendental functions, I, McGraw-Hill, New York-Toronto-London, 1953.

[2] G.B. Folland and E.M. Stein, Estimates for the $\bar{\partial}_{b}$ complex and analysis on the Heisenberg group, Comm. Pure Appl. Math., 27 (1974), 429-522.

[3] D. Geller, Fourier analysis on the Heisenberg group I: Schwartz space, J. Funct. Anal., 36 (1980), 205-254.

[4] Local solvability and homogeneous distributions on the Heisenberg group, Comm. Partial Diff. Eqns., 5 (1980), 475-576.

[5] A. Grossmann and J. Morlet, Decomposition of Hardy functions into squre integrable wavelets of constant shape, SIAM J. Math. Anal., 15 (1984), 723-736.

[6] R. Howe, Quantum mechanics and partial differential equations, J. Funct. Anal., 38 (1980), 188-254.

[7] Q. Jiang and L. Peng, Wavelet transform and Toeplitz-Hankel type operators, Math. Scand., 70 (1992), 247-264.

[8] Toeplitz and Hankel type operators on the upper half-plane, Integral Eqns. Op. Th., 15 (1992), 744-767.

[9] _ Admissible wavelets on the Siegel domain of type one, to appear.

[10] S. Kaneyeki, Homogeneous bounded domains and Siegel domains, Lecture Notes in Math., 241, Springer-Verlag, Berlin-New York, 1971.

[11] A. Korányi and E.M. Stein, $H^{2}$ spaces of general half-planes, Studia Math., 44 (1972), 379-388.

[12] R.D. Ogden and S. Vági, Harmonic analysis of a nilpotent group and function theory on Siegel domains of type II, Adv. in Math., 33 (1979), 31-92.

[13] T. Paul, Functions analytic on the half-plane as quantum mechanical states, J. Math. Phys., 25 (1984), 3252-3263.

[14] W. Rudin, Function theory in the unit ball of $C^{n}$, Springer-Verlag, New YorkHeidelberg-Berlin, 1980.

[15] G. Szegö, Orthogonal polynomials, Amer. Math. Soc. Colloq. Publications, 23, 1939.

[16] N.Ja. Vilenkin, Laguerre polynomials, Whittaker functions and representations of group of bordered matrices, Math. USSR Sb., 4 (1968), 399-410.

Received June 28, 1994 and revised March 31, 1995. The work for this paper was supported by the National Natural Science Foundation of China.

PEKING UNIVERSITY

BeIJING 100871

P.R. CHINA 